\title{
DC Bias Magnetic Vibration Monitoring Method and Device Based on the Amplitude of the Core Transformer
}

\author{
Haokai Xie \\ North China Electric Power University, Beijing, 102206 \\ alanncepu@foxmail.com
}

Keywords: Transformer vibration; DC bias; Finite element method; Monitoring device

\begin{abstract}
In order to overcome the blindness of the installation of sensors in monitoring the vibration of the transformer, in this paper, a method for monitoring the DC bias of transformer based on the amplitude of the iron core is proposed. By establishing the circuit electromagnetic field force field model, a finite element analysis is used to obtain the vibration amplitude of the DC magnetic field at each position of the transformer core, then to collect the low frequency vibration of the core during the operation of the transformer. Analysis of the data and timely warning, can improve the accuracy of the vibration signal acquisition, to avoid the DC bias due to abnormal vibration of the transformer and accident tripping and other major accidents.
\end{abstract}

\section{Introduction}

As one of the most important power equipment in power system, Transformers' health level and running state of power system affect the safe operation of the whole power system. When the local geomagnetic storms occurs, the power grid will be induced by the geomagnetic induction current (GIC), its frequency range of $0.001-0.1 \mathrm{HZ}$, the relative frequency with the $50 \mathrm{HZ}$, is quasi DC [1]. When the GIC flows into the transformer through the neutral point, it will cause the transformer DC bias saturation, produce a large number of harmonics, increase reactive power loss, and makes the transformer vibration, noise increases. Between March 2001 and October 2002, Jiangsu 500kV Yang-Huai transmission system of transformer substation on the river occurred repeatedly due to GIC transformer strong vibration [2, 3]. In March 13, 1989, the Chifeng power plant has occurred GIC caused by the strong vibration of the transformer [4].

The transformer vibration has been studied at home and abroad. However, due to the vibration characteristics of the position of the transformer will be different from the internal structure, the location of the different. At present most of the transformer vibration monitoring methods are faced with the problem that the sensor installation is not clear, cannot show the full transformer vibration.

\section{Vibration Source}

The vibration of transformer mainly includes two parts, the main body vibration and the vibration of the cooling device. The body vibration also includes the vibration of the iron core and the vibration of the winding.

Lvibration of the Iron Core. When the transformer works, the iron core is magnetized and the phenomenon of magnetic occurs. When the magnetic state is changed periodically, the iron core is periodically deformed and the vibration is produced. Magnetostrictive vibration is the main reason for the vibration of the core of the transformer, including other vibration caused by the electromagnetic force between the adjacent silicon steel sheets and the vibration caused by the vibration of the joint between the silicon steel sheet and the vibration caused by the electromagnetic force is also included. But comparing the vibration of the iron core is caused by the magnetostrictive phenomenon; the two parts of the vibration can be ignored [5].

Winding Vibration. The winding vibration is caused by the leakage flux of the transformer and the electromagnetic force generated by the load current. The formula for calculating electromagnetic force is 


$$
\boldsymbol{F}=\oint I d \boldsymbol{l} \boldsymbol{B}
$$

The leakage flux of the transformer will increase under the effect of GIC, caused the electromagnetic force increasing. But domestic and foreign research and test have proved whether it is the normal operation or DC bias, the transformer winding vibration than the magnetic expansion caused by the vibration of the iron core is much smaller, so that the study can be ignored [6].

Cooling Device Vibration. Vibration of the transformer in addition to body vibration, there are cooling device vibration, including the cooling fan, radiator and submersible pumps and other equipment at run time of vibration. The vibration condition is not affected by GIC invasion. In the study it will not be considered and attention should be paid to the screening in the monitoring.

\section{Core Vibration Model}

The key to establishing the core vibration model is to establish the circuit electromagnetic field force field model, The first is to build model calculation to obtain the normal operating voltage of the transformer and the magnetic field of the iron core under the common excitation of GIC, then use the results of vibration displacement of the core of the transformer by calculating to obtained the distribution of the magnetic field. Finite element method and ANSYS finite element method can be introduced to solve the analysis process.

Circuit -electromagnetic Field Model. The model is taking the voltage of the external circuit of the transformer winding as knowing quality, taking the current in the magnetic field of the magnetic core and the winding as the solution. The winding current is solved by the circuit model, and the inductance of the winding is calculated by the magnetic field model, and the alternating iteration circuit and the magnetic field operation are realized.

For some complicated electrical wiring, it can only study the magnetic flux and vibration state of the core under no-load condition, and simplify the circuit. The voltage regulating winding with no load, the exciting winding and the middle and low voltage winding don't play a role, is not in the circuit model. Simulation of transformer operating voltage in the high voltage winding and GIC excitation power supply, That is, an AC voltage source and waveform irregular GIC excitation voltage source is directly connected in series with the primary winding of the transformer.

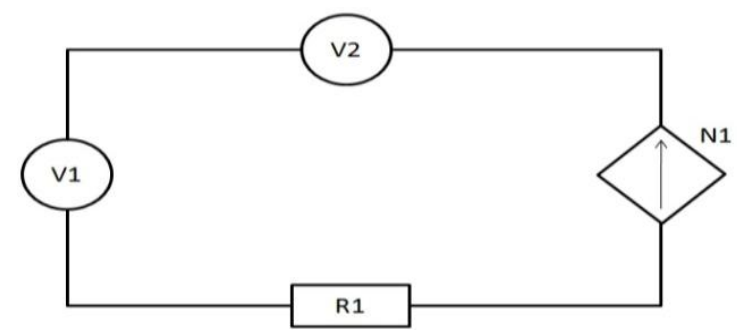

Figure 1. Field circuit coupled model

Fig. 1. Shows the circuit model, V1 represents the independent voltage source of the sine wave, Simulates normal operating voltage of power network. Voltage value is set to the rated voltage of the high voltage side of the transformer.

From the Helmholtz theorem, if know field of curl and divergence and boundary conditions, this field is the only sure. The electric field and the magnetic field divergence and curl satisfies the Maxwell field equations, as: 


$$
\left\{\begin{array}{l}
\nabla \times \boldsymbol{H}=\boldsymbol{J}+\frac{\partial \boldsymbol{D}}{\partial \boldsymbol{t}} \\
\nabla \times \boldsymbol{E}=\frac{\partial \boldsymbol{B}}{\partial \boldsymbol{t}} \\
\nabla \square \boldsymbol{D}=\rho \\
\nabla \square \boldsymbol{B}=\mathbf{O}
\end{array}\right.
$$

With the secondary equation $\mathrm{B}=\mathrm{H}$ and $\mathrm{D}=\mathrm{E}$ substitution, The derivation and finishing process to obtain the magnetic vector a and the field source current density $\mathrm{J}$, scalar potential phi and field source charge density $\mathrm{p}$, the expression of relationship between them is as follows:

$$
\begin{aligned}
& \nabla^{2} \mathrm{~A}=-\mu \mathrm{J} \\
& \nabla^{2} \varphi-\mu \varepsilon \frac{\partial^{2} \varphi}{\partial \mathrm{t}^{2}}=-\frac{\rho}{\varepsilon}
\end{aligned}
$$

Due to the magnetic permeability of the core with respect to the wire, air and insulating oil is equivalent to infinity, the model need be solved with the boundary conditions: The external surface of the iron core is parallel to the outer surface of the core. Using the finite element method, the distribution of the magnetic potential field is obtained, the magnetic field strength can be obtained.

Electromagnetic Field-Force Field Model. Taking the magnetic induction intensity obtained to calculate magnetostrictive force into the dynamic characteristic equation can be obtained the vibration displacement of the core. In study, according to the transformer iron core laminated structure and magnetic field lines along which the core parallel distribution characteristics, the space stress problem is simplified as plane force problem.

The transformer is decomposed into a finite element. The vibration of the core of the transformer is the process of the periodic displacement of each small unit under the combined action of internal and external forces. the external force includes the magnetostrictive force, electromagnetic force and gravity force. Internal force is the internal stress when the core is deformed. The force acting on the object is the stress, and the deformation of the object when the object is subjected to the external force is a strain. The schematic diagram of the strain and stress is shown in the figure below.
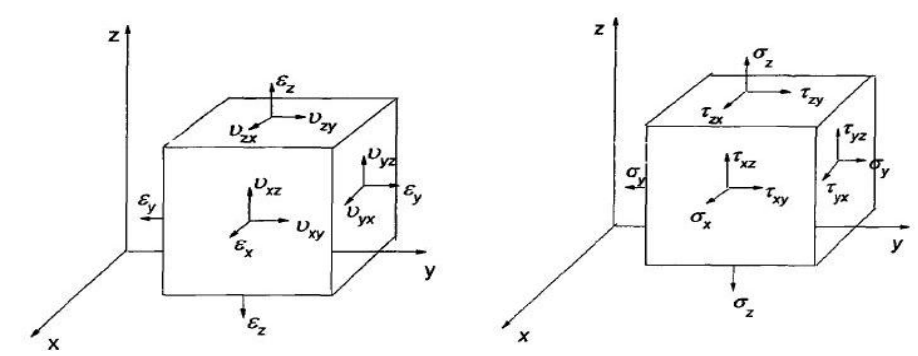

Figure 2. Strain and stress component diagram

The relationship between stress, strain and external force is described as follows

Equilibrium equation of stress and external force

$$
\begin{aligned}
& \left\{\begin{array}{l}
\frac{\partial \sigma_{\mathrm{x}}}{\partial \mathrm{x}}+\frac{\partial \tau_{\mathrm{yx}}}{\partial \mathrm{y}}+\frac{\partial \tau_{\mathrm{zx}}}{\partial \mathrm{z}}+\mathrm{P}_{\mathrm{x}}=\mathrm{m} \frac{\partial^{2} \mathrm{u}}{\partial \mathrm{x}^{2}} \\
\frac{\partial \tau_{\mathrm{xy}}}{\partial \mathrm{x}}+\frac{\partial \sigma_{\mathrm{y}}}{\partial \mathrm{y}}+\frac{\partial \tau_{\mathrm{zy}}}{\partial \mathrm{z}}+\mathrm{P}_{\mathrm{y}}=\mathrm{m} \frac{\partial^{2} \mathrm{v}}{\partial \mathrm{y}^{2}} \\
\frac{\partial \tau_{\mathrm{xz}}}{\partial \mathrm{x}}+\frac{\partial \tau_{\mathrm{yz}}}{\partial \mathrm{y}}+\frac{\partial \sigma_{\mathrm{z}}}{\partial \mathrm{z}}+\mathrm{P}_{\mathrm{z}}=\mathrm{m} \frac{\partial^{2} \mathrm{w}}{\partial \mathrm{z}^{2}}
\end{array}\right. \\
& \{P\}=\left\{P_{x} P_{y} P_{z}\right\} \text { is external force for an object. }
\end{aligned}
$$

The expression of magnetostrictive force is [7] : 
$\mathrm{F}_{\mathrm{ms}}^{\mathrm{e}}=-\int\left[\left(\mathrm{B}_{\mathrm{x}}^{\mathrm{e}}\right)^{2} \frac{\mathrm{E}}{(1+\alpha)(1-2 \alpha)}\right] \mathrm{dxdy}-\int\left[\left(\mathrm{B}_{\mathrm{y}}^{\mathrm{e}}\right)^{2} \frac{\mathrm{E}}{(1+\alpha)(1-2 \alpha)}\right] \mathrm{dxdy}$

The expression of the unit electric power is [8]:

$$
F_{\mathrm{mw}}^{\mathrm{e}}=\int\left[\mathrm{v}_{\mathrm{o}}(\mathbf{n} \sqcap \mathbf{B}) \mathbf{B}-\frac{1}{2} \mathrm{v}_{\mathrm{o}} \mathbf{B}^{2} \mathbf{n}\right] \mathrm{dxdy}
$$

Relationship between stress and strain:

$$
\left\{\begin{array}{c}
\varepsilon_{x}=\frac{1}{E}\left(\sigma_{x}-\mu \sigma_{y}\right) \\
\varepsilon_{y}=\frac{1}{E}\left(\sigma_{y}-\mu \sigma_{x}\right) \\
\varepsilon_{z}=\frac{\mu}{E}\left(\sigma_{x}-\sigma_{y}\right) \\
\gamma_{x y}=\frac{1}{G} \tau_{x y}
\end{array}\right.
$$

Relationship between strain and particle displacement:

$$
\{\varepsilon\}=\left\{\begin{array}{c}
\varepsilon_{\mathrm{x}} \\
\varepsilon_{\mathrm{y}} \\
\gamma_{\mathrm{xy}}
\end{array}\right\}=\left\{\begin{array}{c}
\frac{\partial \mathbf{u}}{\partial \mathrm{x}} \\
\frac{\partial \mathbf{v}}{\partial \mathbf{y}} \\
\frac{\partial \mathbf{u}}{\partial \mathrm{y}}+\frac{\partial \mathbf{v}}{\partial \mathrm{x}}
\end{array}\right\}
$$

The external force is known, the stress, the strain and the displacement are unknown, multiple (1) (2) (3) in the equation, using displacement method [9] to get solution.

After solving the displacement values of each unit, the vibration displacement of any point is determined according to the interpolation method. At this time, it should be assumed that the interpolation function can be polynomial:

$$
\left\{\begin{array}{l}
\mathrm{u}(\mathrm{x}, \mathrm{y})=\alpha_{1}+\alpha_{2} \mathrm{x}+\alpha_{3} \mathrm{y} \\
\mathrm{v}(\mathrm{x}, \mathrm{y})=\alpha_{4}+\alpha_{5} \mathrm{x}+\alpha_{6} \mathrm{y}
\end{array}\right.
$$

At the same time, considering that the bottom of the core is fixed, the displacement is zero and the other boundary is free boundary condition.

\section{Model Instance}

Taking the actual parameters [10] of the ODFPS-500000/750 three phase transformer in Northwest $750 \mathrm{kV}$ UHV power grid into model can obtain the core vibration displacement. This type of transformer vibration displacement maximum position appears on the iron yoke surface connecting the two column and the yoke iron core column and column side surface connection. The vibration displacement of the bottom side of the transformer iron core is the smallest due to the base of the fixed effect of the iron core.

\section{Design of the Monitoring Device}

Study shows that the inherent frequency of the transformer core between above $250 \mathrm{HZ}$ [11], below $200 \mathrm{HZ}$, the vibration frequency will not be due to the inherent vibration frequency of the core and resonance, whose signal characteristic of the core can be more truly reflected the GIC vibration of the core. Therefore, in this paper, a new type of DC bias magnetic vibration monitoring device based on the amplitude of the iron core is proposed. 
The technical scheme of the device is: the monitoring position of the iron core amplitude is obtained through the model analysis, and the sensor is arranged at the corresponding position on the surface of the oil tank. The vibration signal of the transformer body is collected by the acceleration sensor placed on the wall of the oil tank wall of the transformer, after the amplifier processing, and then through the data acquisition card into the analysis of industrial control. By analyzing the variation characteristics of the signal, the time and the influence degree of the DC bias magnetic field are given, and the alarm signal is sent out when the operation state of the transformer is distorted. The utility model has the advantages that the vibration of the different position of the transformer can be collected, and the prioritized collected larger signal amplitude transformer core. Using this equipment to monitor and analyze transformer vibration, steps are as follows:

1. When there is no DC bias, monitor the low frequency vibration of the transformer in a certain period of $200 \mathrm{HZ}$, in order to record the characteristic data of transformer vibration when the monitoring point is not DC biased. The frequency spectrum of the vibration signal of the iron core only comprises a $100 \mathrm{HZ}$ and an integer frequency of the vibration component.

2. When the DC bias occurs, it will be able to observe the change trend of the apparent and continuous. Frequency spectrum comes $50 \mathrm{HZ}, 150 \mathrm{HZ}, 50 \mathrm{HZ}$, and other odd times the frequency of the vibration component. At this time the device gives warning signal

3. Device accords to the phase of $50 \mathrm{HZ}$ and $150 \mathrm{HZ}$ vibration components in the vibration signal spectrum to determine the cause of DC magnetic bias vibration.

1).The judgement of Substantial phase change is caused by the role of GIC;

2).The judgement of Phase change which is not large is caused by HVDC input current;

3).If the long-term warning of the situation, it needs a further detection of the transformer body signal (doubt the internal structure of the transformer may be a problem). Otherwise, the operation condition of the transformer is considered to keep good.

\section{Conclusion}

When the magnetic storm occurs, GIC intrusion transformer will cause the transformer vibration, and the vibration signal spectrum signal in the odd number of $50 \mathrm{HZ}$ components, its amplitude increases with the increase of GIC.

The transformer DC magnetic bias vibration is mainly caused by the vibration of the iron core. Based on the analysis to get the distribution of the vibration displacement of the core, which can be more effective in monitoring the vibration of the transformer.

\section{References}

[1] Kappenman J G, Albertson V D. Bracing for the Geomagnetic Storms [J]. IEEE Spectrum, 1990, 27(3):27-33

[2] Liu Linyu, Xie Xuewu. Abnormal sound analysis of main transformer in 500kV [J].High voltage technology, 2005, 31 (4): 85-87

[3] Kuai Dizheng, Liu Chengmin, Wanda. Study on the influence of DC bias on the [J]. Jiangsu electrical engineering, 2004, 23 (3): 1-5

[4] Liu Lianguang, Liu Chunming, Zhang Bing, et al. several strong magnetic storm events of China Guangdong power grid [J].Chinese Journal of Geophysics, 2008,51 (4): 976-981

[5] Gu Xiaoan, Shen Rongying, Xu Jitai. Research on vibration and noise control methods of large power transformer [J]. Noise and vibration control, 2001, 5:7-11

[6] Picher P, Bolduc L, Dutil A, et al. Study of the acceptable DC current limit in core-form power transformers [J]. IEEE transactions on Power Systems, 1997, 12(1):194-207

[7] Mohammed O A, Liu S, Abed N. Study of the Inverse Magnetostriction Effect on Machine Deformation[C]. IEEE SoutheastCon, 2004:433-436 
[8] Yan Weili, Yang Qingxin, Wang Youhua. Numerical analysis of electromagnetic field in electrical engineering [M]. Beijing: Mechanical Industry Press, 2005

[9] Du Wancheng. Step by step calculation of displacement method of finite element method [J]. Journal of Chongqing Architectural University, 1979, 1:011

[10]Pei Xiaoran. Research on vibration characteristics and monitoring methods of three phase transformer [D]. GIC Beijing: North China Electric Power University, 2015 